\title{
Análisis del clima y cultura organizacional en el Hospital de Tabio: caso de estudio ${ }^{1}$
}

\section{Analysis of the organizational climate and culture in the Tabio Hospital case study}

\author{
Luz Deicy Flórez ${ }^{2}$ \\ Eduar Alberto Mora Vergara \\ DOI: 10.29151/hojasyhablas.n18a8
}

\section{Resumen}

El Hospital Nuestra Señora del Carmen en Tabio (Cundinamarca) está catalogado como un hospital público de primer nivel. Con los años, se ha desarrollado en infraestructura y tecnología y ha ido incorporando nuevas especialidades acordes con las necesidades de sus usuarios. En la actualidad, la organización cuenta con dos tipos de personal; por un lado, quienes trabajan directamente en la práctica misional, tales como médicos, enfermeras, psicólogos, camilleros, etc., y quienes brindan apoyo en las áreas administrativa, vigilancia y de servicios generales. La institución ha venido presentando problemas relacionados con la mala comunicación, la pérdida de interés de sus colaboradores y la falta de interés por los programas de gestión de talento humano, con consecuencias negativas en el logro de los objetivos organizacionales. Con la presente investigación, se revisó el componente de Gestión del Talento Humano y se realizó un estudio del clima y cultura organizacional, para identificar los factores problemáticos más preponderantes del Hospital, y sugerir estrategias de mejoramiento. Con los resultados del análisis de clima y cultura organizacionales se evidenciaron los factores que alteran el ambiente laboral, las variables que generan discordia entre empleados, y las claves para lograr mejorar el ambiente laboral. Dentro de las principales conclusiones, se encontraron deficiencias en la comunicación interna al momento de dar a transmitir decisiones estratégicas; adicionalmente se identificó un sistema de contratación desigual, resistencia al cambio y falta de estabilidad laboral.

Palabras clave: Gestión hospitalaria; clima organizacional; cultura organizacional; talento humano.

\begin{abstract}
The Nuestra Señora del Carmen Hospital is listed as a first level public hospital. Over the years, it has developed in infrastructure and technology and has been incorporating new specialties according to the needs of its users. Currently, the administrative organization has two types of staff; on the one hand, there are doctors, nurses, psychologists, stretchers, etc., and on the other hand, employees who provide support from the administrative areas, surveillance and general services. As a result, the institution has been presenting problems related to poor communication, loss of interest of its employees and lack of interest in human talent management programs, with negative consequences in the achievement of organizational objectives. This paper shows a review of Human Talent Management component for the Tabio Hospital, and within that analysis, carry out a climate and organizational culture study to identify the most preponderant problematic factors of the Hospital, and suggest improvement strategies. With the results of the analysis of organizational climate and culture, it was possible to demonstrate the factors that alter the work environment, the variables that generate discord among employees, and the keys to improve the work environment. Among the main conclusions, deficiencies in internal communication at the time of making strategic decision. Additionally, an unequal accounting system, resistance to change, and lack of job stability were identified.
\end{abstract}

Keywords: Hospital management; organizational climate; organizational culture; human talent.

${ }^{1}$ Artículo de investigación resultado de la tesis de pregrado de Administración de Empresas titulada "Propuesta metodológica para medir la cultura y el clima organizacional del Hospital Nuestra Señora del Carmen de Tabio, Cundinamarca" para el proyecto de investigación "Desarrollo de herramientas de software y técnicas para el apoyo de los procesos de gestión y administración en el Hospital de Tabio-Componente de Gestión de Talento Humano", financiado con el Fondo de Investigaciones de Universidad de Cundinamarca para la Extensión Chía.

${ }^{2}$ Investigadora Universidad de Cundinamarca Extensión Chía, Grupo de Investigación DOPYS. E-mail: ldeicyflorez@ucundinamarca.edu.co. ORCID: https://orcid.org/0000-00016186-656X

${ }^{3}$ Integrante del Semillero de Investigación RH-PRO de la Universidad de Cundinamarca Extensión Chía. E-mail: eamora@ucundinamarca.edu.co.

100 H HAS Y HABLAS 


\section{Introducción}

El Hospital de Tabio solicitó a la Universidad de Cundinamarca un análisis de gestión hospitalaria dentro del cual se pudo adelantar un proyecto de investigación para el diseño de una propuesta de análisis de clima y cultura organizacional. Dentro de la investigación se pudo conocer la percepción de los colaboradores y analizar la situación actual mediante una metodología cualitativa que incluyó métodos como la observación, la aplicación de encuestas y la realización de entrevista con los empleados del Hospital.

Los resultados más relevantes indicaron una resistencia de los empleados del Hospital Nuestra Señora del Carmen en cuanto a la forma de contratación, ya que se combinan nombramientos de empleados entre modalidades de prestación de servicios y contratistas, lo que genera molestias e insatisfacción en algunas áreas de la organización.

Los resultados de la investigación le permitieron al Hospital implementar nuevas acciones estratégicas, con el objetivo de tener un personal motivado, comprometido con los objetivos, misión y visión de la entidad, y afianzar procesos de comunicación asertiva para impulsar un mejor clima laboral.

\section{Metodología}

La metodología elegida para la investigación fue de carácter cualitativo, puesto que la información se recolectó a partir de la observación de comportamientos naturales, discursos, respuestas a entrevistas y datos recolectados en estudios anteriores realizados por la Institución. El proceso se desarrolló en tres fases: 1) Recolección de información; 2) Análisis de información y 3) Diseño de propuesta. Para la recolección de la información referente a la cultura organizacional se utilizaron dos instrumentos diseñados con base en los modelos planteados por Denison y Neale (citados por Bonavida y Prado, 2010) en la medición de la cultura organizacional. Así mismo, en la medición del clima, se utilizó el modelo presentado por la Cámara de Comercio de Barranquilla (2015).

Con estos dos modelos, se llevó a cabo el análisis de la situación actual de los colaboradores del Hospital con relación a las áreas de facturación, farmacia, hospitalización, medicina general, servicios generales, subgerencias y gerencia general. El estudio se en consideración las cuatro variables de las que trata el instrumento de Denison (2000), las cuales son: Implicación, Consistencia, Adaptabilidad y Misión (Bonavida y Prado, 2010, p.p. 17-19).

\section{Figura No. 1. Variables consideradas en el estudio de Cultura Organizacional para el Hospital de Tabio}

\begin{tabular}{|c|c|}
\hline Implicación & Consistencia \\
\hline$\checkmark$ Empoderamiento & Valores centrales \\
\hline$\checkmark \quad$ Trabajo en equipo & Acuerdos \\
\hline$\checkmark \quad$ Desarrollo de capacidades & $\checkmark \quad$ Coordinación e integración \\
\hline Adaptabilidad & Misión \\
\hline$\checkmark$ Orientación al cambio & $\checkmark$ Dirección y propósito estratégico \\
\hline$\checkmark \quad$ Orientación al cliente & $\checkmark \quad$ Metas y objetivos \\
\hline$\checkmark$ Aprendizaje organizativo & $\checkmark \quad$ Visión \\
\hline
\end{tabular}

Fuente: Bonavida y Prado, 2010, p.p. 17-19

Una vez determinadas las variables y sus factores, se aplicó el siguiente cuestionario que tiene un sistema de respuesta binario (Si o No), lo anterior como sugerencia del Hospital con el objetivo de obtener un análisis asertivo de la medición de la cultura organizacional. 
Tabla No. 1. Cuestionario de medición de la cultura organizacional.

\begin{tabular}{|c|c|c|}
\hline \multirow{2}{*}{$\begin{array}{l}\text { Características o variables } \\
\text { IMPLICACION }\end{array}$} & $\mathrm{Si}$ & No \\
\hline & & \\
\hline 1. La mayoría de los miembros de este grupo están muy comprometidos con su trabajo. & & \\
\hline $\begin{array}{l}\text { 2. Las decisiones con frecuencia se toman en el nivel que dispone de la mejor } \\
\text { información. }\end{array}$ & & \\
\hline 3. La información se comparte ampliamente y se puede conseguir la que se requiere. & & \\
\hline 4. Cada miembro cree que puede tener un impacto positivo en el grupo. & & \\
\hline 5. La planificación de nuestro trabajo es continua e involucra a todo el equipo. & & \\
\hline $\begin{array}{l}\text { 6. Se fomenta activamente la cooperación entre los diferentes grupos de esta } \\
\text { organización. }\end{array}$ & & \\
\hline 7. Trabajar en este grupo es como formar parte de un equipo & & \\
\hline $\begin{array}{l}\text { 8. Acostumbramos a realizar las tareas en equipo, en vez de descargar el peso en la } \\
\text { dirección. }\end{array}$ & & \\
\hline 9. Los grupos y no los individuos son los principales pilares de la organización. & & \\
\hline $\begin{array}{l}\text { 10. El trabajo se organiza de modo cada persona entiende la relación entre su trabajo y } \\
\text { los objetivos. }\end{array}$ & & \\
\hline 11. La autoridad se delega de modo que las personas puedan actuar por sí mismas. & & \\
\hline $\begin{array}{l}\text { 12. Las capacidades del "banquillo" (los futuros líderes en el grupo) se mejoran } \\
\text { constantemente. }\end{array}$ & & \\
\hline $\begin{array}{l}\text { 13. Este grupo invierte continuamente en el desarrollo de las capacidades de sus } \\
\text { miembros. }\end{array}$ & & \\
\hline $\begin{array}{l}\text { 14. La capacidad de las personas es vista como una fuente importante de ventaja } \\
\text { competitiva. }\end{array}$ & & \\
\hline $\begin{array}{l}\text { 15. A menudo surgen problemas porque no disponemos de las habilidades necesarias para } \\
\text { hacer el trabajo. }{ }^{*}\end{array}$ & & \\
\hline CONSISTENCIA & & \\
\hline 16. Los líderes y directores practican lo que pregonan. & & \\
\hline 17. Existe un estilo de dirección característico con un conjunto de prácticas distintivas. & & \\
\hline $\begin{array}{l}\text { 18. Existe un conjunto de valores claro y consistente que rige la forma en que nos } \\
\text { conducimos. }\end{array}$ & & \\
\hline 19. Ignorar los valores esenciales de este grupo te ocasionará problemas. & & \\
\hline $\begin{array}{l}\text { 20. Existe un código ético que guía nuestro comportamiento y nos ayuda a distinguir lo } \\
\text { correcto. }\end{array}$ & & \\
\hline $\begin{array}{l}\text { 21. Cuando existen desacuerdos, trabajamos intensamente para encontrar soluciones } \\
\text { donde todos ganen. }\end{array}$ & & \\
\hline 22. Este grupo tiene una cultura "fuerte". & & \\
\hline 23. Nos resulta fácil lograr el consenso, aun en temas dificiles. & & \\
\hline 24. A menudo tenemos dificultades para alcanzar acuerdos en temas claves. & & \\
\hline 25. Existe un claro acuerdo acerca de la forma correcta e incorrecta de hacer las cosas. & & \\
\hline 26. Nuestra manera de trabajar es consistente y predecible. & & \\
\hline 27. Las personas de diferentes grupos de esta organización tienen una perspectiva común. & & \\
\hline 28. Es sencillo coordinar proyectos entre los diferentes grupos de esta organización. & & \\
\hline $\begin{array}{l}\text { 29. Trabajar con alguien de otro grupo de esta organización es como trabajar con alguien } \\
\text { de otra organización. }\end{array}$ & & \\
\hline
\end{tabular}


30. Existe une buena alineación de objetivos entre los diferentes niveles jerárquicos.

ADAPTABILIDAD

31. La forma que tenemos de hacer las cosas es flexible y fácil de cambiar.

32. Respondemos bien a los cambios del entorno.

33. Adoptamos de continuo nuevas y mejores formas de hacer las cosas.

34. Los intentos de realizar cambios suelen encontrar resistencias.

35. Los diferentes grupos de esta organización cooperan a menudo para introducir cambios.

36. Los comentarios y recomendaciones de nuestros clientes conducen a menudo a introducir cambios.

37. La información sobre nuestros clientes influye en nuestras decisiones.

38. Todos tenemos una comprensión profunda de los deseos y necesidades de nuestro entorno.

39. Nuestras decisiones ignoran con frecuencia los intereses de los clientes.

40. Fomentamos el contacto directo de nuestra gente con los clientes.

41. Consideramos el fracaso como una oportunidad para aprender y mejorar.

42. Tomar riesgos e innovar son fomentados y recompensados.

43. Muchas ideas "se pierden por el camino".

44. El aprendizaje es un objetivo importante en nuestro trabajo cotidiano.

45. Nos aseguramos que "la mano derecha sepa lo que está haciendo la izquierda".

MISION

46. Esta organización tiene un proyecto y una orientación a largo plazo.

47. Nuestra estrategia sirve de ejemplo a otras organizaciones.

48. Esta organización tiene una misión clara que le otorga sentido y rumbo a nuestro trabajo.

49. Esta organización tiene una clara estrategia de cara al futuro.

50. La orientación estratégica de esta organización no me resulta clara.

51. Existe un amplio acuerdo sobre las metas a conseguir.

52. Los líderes y directores fijan metas ambiciosas pero realistas.

53. La Dirección nos conduce hacia los objetivos que tratamos de alcanzar.

54. Comparamos continuamente nuestro progreso con los objetivos fijados.

55. Las personas de esta organización comprenden lo que hay que hacer para tener éxito a largo plazo.

56. Tenemos una visión compartida de cómo será esta organización en el futuro.

57. Los líderes y directores tienen una perspectiva a largo plazo.

58. El pensamiento a corto plazo compromete a menudo nuestra visión a largo plazo

59. Nuestra visión genera entusiasmo y motivación entre nosotros.

60. Podemos satisfacer las demandas a corto plazo sin comprometer nuestra visión a largo plazo.

Fuente: Bonavida y Prado, 2010, p.p. 21-23. 
De otro lado, en cuanto a la investigación sobre clima organizacional, se utilizó el formulario propuesto por la Cámara de Comercio de Barranquilla (2015), el cual fue diseñado a partir de una investigación realizada en empresas afiliadas, lo que otorga rigor y garantiza que las variables estudiadas sean las adecuadas, especialmente para el caso de empresas colombianas. El instrumento cuenta con 47 preguntas agrupadas en 8 características, con un amplio rango de información.

\section{Tabla No. 2. Variables para medición de clima organizacional}

\begin{tabular}{|c|c|c|}
\hline \multicolumn{3}{|l|}{ VARIABLES } \\
\hline AMBIENTE LABORAL & SI & NO \\
\hline 1.Me siento muy satisfecho con mi ambiente de trabajo & & \\
\hline 2. En mi organización está claramente definida su Misión y Visión & & \\
\hline 3. Cuenta con los materiales y equipo necesario para realizar su trabajo. & & \\
\hline 4. Salgo del trabajo sintiéndome satisfecho de lo que he hecho & & \\
\hline 5. En esta organización valoran mi trabajo & & \\
\hline 7. Estoy satisfecho con los beneficios que recibo por parte de la organización & & \\
\hline AUTONOMIA & & \\
\hline 8. Mi superior me motiva a cumplir con mi trabajo del a manera que yo considere mejor & & \\
\hline 9. Mi superior me motiva a cumplir con mi trabajo de la manera que yo mejor considere & & \\
\hline 10. Soy responsable de cumplir los estándares de desempeño y/o rendimiento & & \\
\hline 11. Conozco las exigencias de mi trabajo & & \\
\hline 12. Me siento comprometido para alcanzar las metas establecidas. & & \\
\hline 13. El horario de trabajo me permite atender mis necesidades personales & & \\
\hline COHESIÓN & & \\
\hline 14. Mis compañeros y yo trabajamos juntos de manera efectiva. & & \\
\hline $\begin{array}{l}\text { 15. En mi grupo de trabajo, solucionar el problema es más importante que encontrar algún } \\
\text { culpable }\end{array}$ & & \\
\hline 16. Mi superior inmediato toma acciones que refuerzan el objetivo común de la Institución. & & \\
\hline 17. Mi superior inmediato pide mis opiniones para ayudarle a tomar decisiones & & \\
\hline $\begin{array}{l}\text { 18. Mi superior inmediato busca los aportes del equipo para que se puedan comprender y } \\
\text { dar solución a las actitudes complejas que se presentan. }\end{array}$ & & \\
\hline 19. Mi superior inmediato me exhorta a mejorar mis capacidades o educación. & & \\
\hline 20. Mi superior inmediato posee una clara visión de la dirección de nuestro grupo de trabajo. & & \\
\hline PRESION & & \\
\hline 21. Tengo mucho trabajo y poco tiempo para realizarlo. & & \\
\hline 22. Mi institución es un lugar relajado para trabajar. & & \\
\hline $\begin{array}{l}\text { 23. En casa, a veces temo oír sonar el teléfono porque pudiera tratarse de alguien que llama } \\
\text { sobre un problema en el trabajo. }\end{array}$ & & \\
\hline 24. Me siento como si nunca tuviese un día libre. & & \\
\hline $\begin{array}{l}\text { 25. Muchas de los trabajadores de mi empresa a mi nivel, sufren de un alto estrés, debido a } \\
\text { la exigencia de trabajo. }\end{array}$ & & \\
\hline $\begin{array}{l}\text { 26. Para desempeñar las funciones de mi puesto tengo que hacer un esfuerzo adicional y } \\
\text { retador en el trabajo }\end{array}$ & & \\
\hline APOYO & & \\
\hline $\begin{array}{l}\text { 27. Hay evidencia de que mi jefe me apoyo utilizando mis ideas o propuestas para mejorar } \\
\text { el trabajo. }\end{array}$ & & \\
\hline 28. Considero que mi jefe es flexible y justo ante las peticiones que solicito. & & \\
\hline
\end{tabular}




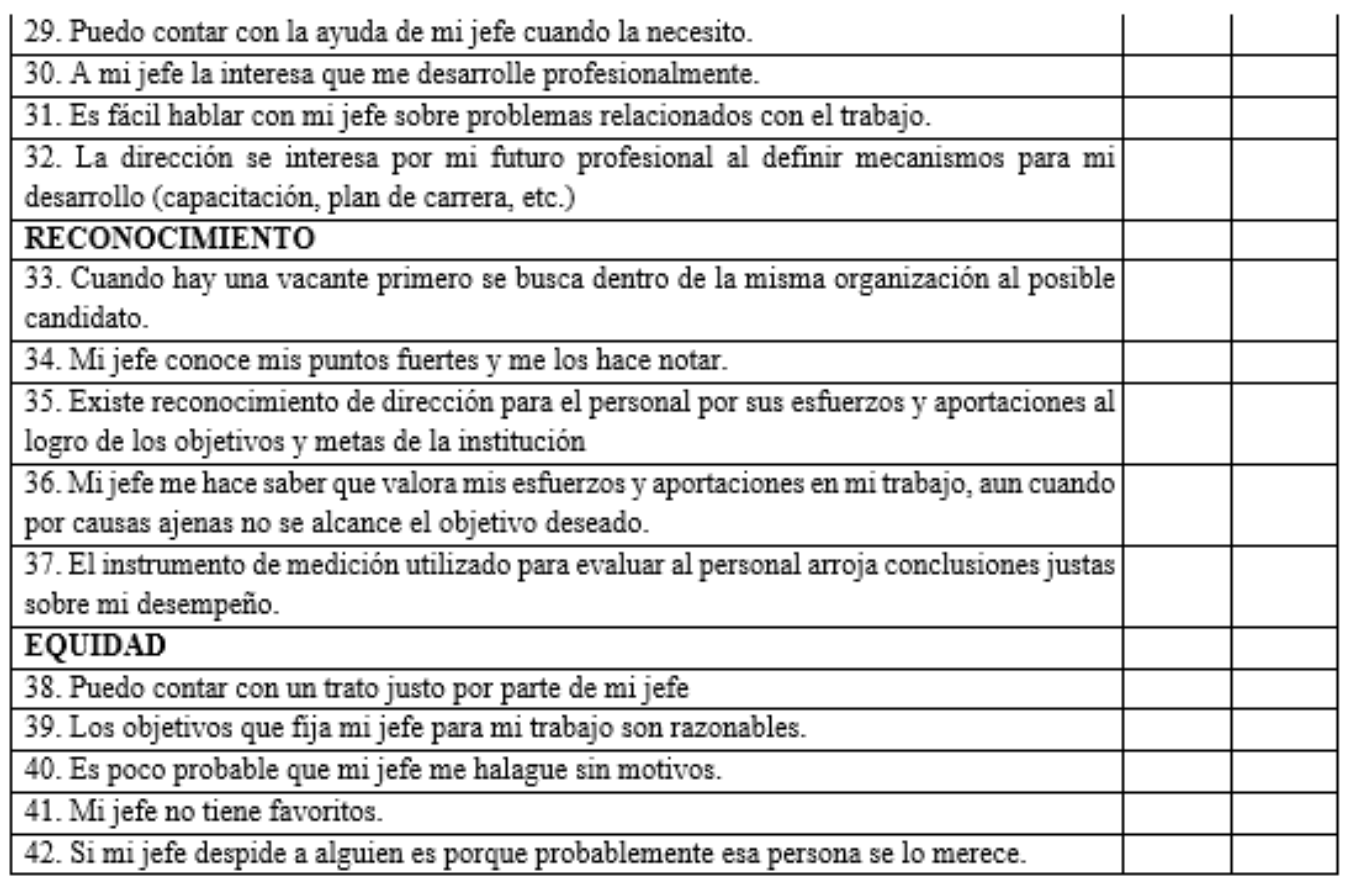

Fuente: Cámara De Comercio De Barranquilla (2015).

\section{Resultados y discusión}

Los instrumentos de medición se diseñaron en formularios electrónicos y fueron remitidos al correo electrónico de cada empleado del Hospital. Adicionalmente, mediante comunicados por parte de la Gerencia General y de la Coordinación de Talento Humano, se solicitó su diligenciamiento en forma anónima. Únicamente el 30\% de los empleados diligenció la información, lo cual evidenció una apatía de los trabajadores frente al diseño de actividades en materia de Gestión del Talento Humano. Por tratarse de una investigación con metodología cualitativa, el 70\% de silencio obtenido en las respuestas, se tomó como uno de los resultados de evaluación y análisis. De esta manera, la investigación de aplicación de los instrumentos de cultura y clima organizacional, se complementó con la aplicación de entrevistas y la realización de talleres en temas de gestión de talento humano.
A continuación, se presentan los datos obtenidos mediante los instrumentos:

\section{Análisis de la cultura organizacional}

Variable 1-Implicación: la primera variable se analizó con 15 ítems del instrumento obteniendo los siguientes resultados:

- Quienes respondieron a esta variable se encuentran muy comprometidos con el trabajo.

- Las decisiones con frecuencia se toman en el nivel que dispone de la mejor información.

- La información se comparte ampliamente y lo que se necesita, se puede encontrar fácilmente.

- Cada miembro cree que puede tener un impacto positivo en el grupo.

- Se fomenta activamente la cooperación entre los diferentes grupos de esta organización.

- Al trabajar, se forma parte de un equipo.

- Se acostumbra a realizar las tareas en equipo, en vez de descargar el peso en la dirección. 
- Los grupos y no los individuos son los principales pilares de esta organización.

- El trabajo se organiza de modo que cada persona entiende la relación entre su trabajo y los objetivos de la organización.

- La autoridad se delega con el fin de que las personas puedan actuar por sí mismas.

- Las capacidades del "banquillo" (los futuros líderes en el grupo) se mejoran constantemente.

- Este grupo invierte continuamente en el desarrollo de las capacidades de sus miembros.

- La capacidad de las personas es vista como una fuente importante de ventaja competitiva.

Una síntesis de los resultados obtenidos, y su relación con la cultura organizacional, se puede observar en el siguiente gráfico:

Figura No. 2. Resultados de la variable Implicación.

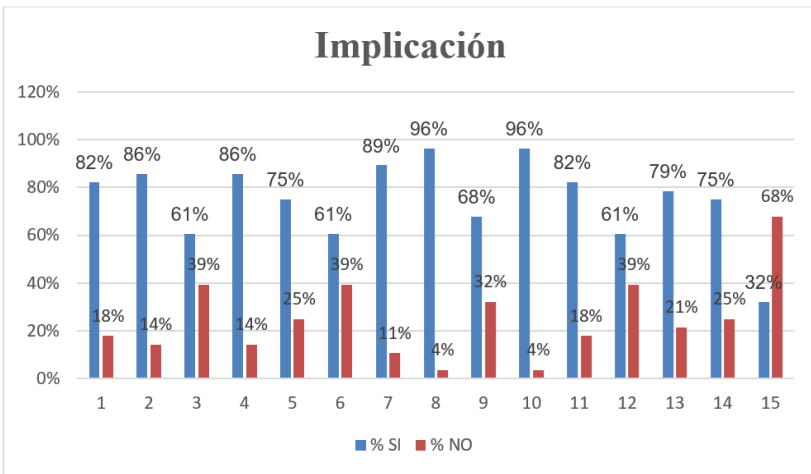

Fuente: Elaboración propia.

Con los datos obtenidos, se observa que los colaboradores del Hospital están comprometidos con su trabajo, es decir que, tienen sentido de pertenecía. También manifiestan que se sienten a gusto con el ambiente laboral y las condiciones en las que desempeñan sus funciones. El nivel de empoderamiento se calcula en el $86 \%$, lo que se considera alto y demuestra que se comprometen, al sentir satisfacción por lo que hacen, y al tomar / hacer parte de las decisiones al momento de realizar o cumplir objetivos; en contraposición con el $14 \%$ que no están de acuerdo y consideran que solo están realizando una labor para la que han sido contratados.
En cuanto al trabajo en equipo, el $96 \%$ de los encuestados manifestaron trabajar en grupo. El restante 4\%, que corresponde a solo una persona, está en desacuerdo con la forma como se orienta el trabajo para alcanzar los objetivos de la organización. El tercer aspecto: desarrollo de capacidades, se evidencia que el $68 \%$ de los entrevistados siente que no cuenta con las herramientas necesarias para realizar un trabajo colaborativo con áreas distintas a la suya; y esto es una percepción que se puede considerar normal, puesto que se trata de un trabajo clínico que requiere un nivel de competencias y conocimientos muy específico.

\section{Figura No. 3. Resultados de la variable Consistencia.}

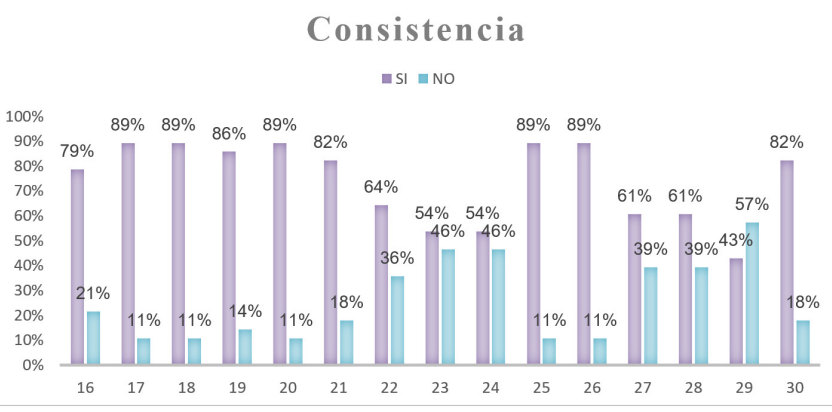

Fuente: Elaboración propia

Los datos obtenidos en la variable Consistencia muestran que, el $86 \%$ de quienes respondieron el instrumento tiene conocimiento de los manuales de funciones, código ético, principios y normas de cada una de las áreas de trabajo; contrario a un $14 \%$ que manifiestan no conocerlos, lo que dificulta el desarrollo normal del trabajo.

Por su parte, la variable 3: Adaptabilidad comprende los ítems del 31 a 45 con tres factores -orientación al cambio, enfoque al cliente y aprendizaje organizacional. En el caso del Hospital de Tabio, este proceso de adaptabilidad se toma como guía e implica asumir riesgos, aprendizaje y una integración de los cambios a los diferentes procesos de la organización con el fin de mejorar las condiciones de las diferentes áreas de trabajo. En la tabulación se encontró la siguiente información: 
Figura No. 4. Resultados de la variable Adaptabilidad.

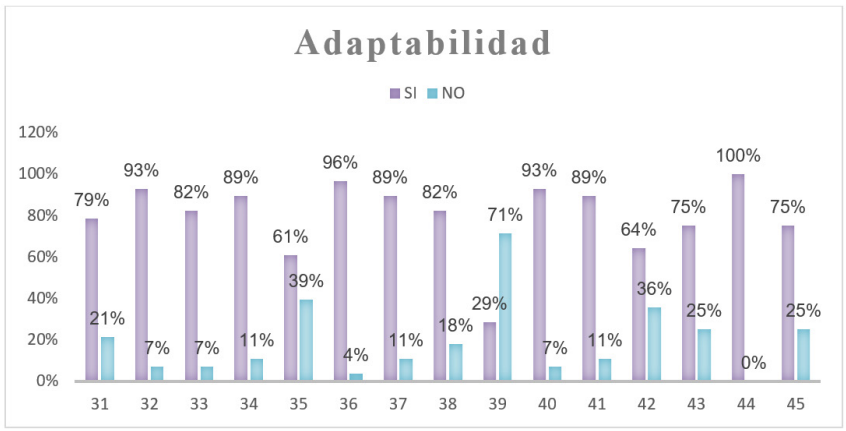

Fuente: Elaboración propia

En el proceso de adaptabilidad, el 93\% de las personas consultadas manifestaron que la organización cuenta con un nivel alto de adaptabilidad a las condiciones de contratación y la forma de asumir el trabajo. En contraste, un 7\% -que representa 2 a 3 personas-, se muestran inconformes con las diferentes condiciones que hay entre las personas nombradas y las que tienen contrato de prestación de servicios. En este sentido, se muestra que un $71 \%$ de las decisiones organizacionales se toman sin tener en cuenta al cliente. Sin embargo, un $29 \%$ considera que el cliente es importante para el Hospital.

Variable 4: Misión: la última variable analizada es la misión, que presenta tres factores 1) Dirección y propósito estratégico, 2) Metas y objetivos y 3) Visión, medido a través de los ítems del 46 al 60. Así, al realizar el cuestionario se obtuvieron los siguientes resultados:

Figura No. 5. Resultados de la variable Misión.

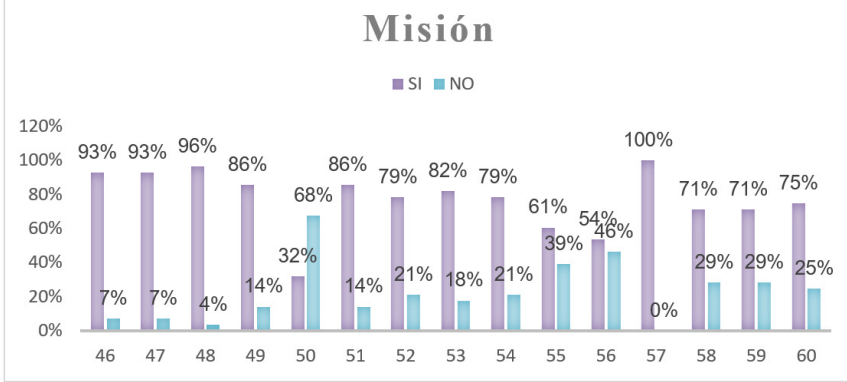

Fuente: Elaboración propia
El 68\% de los encuestados están en desacuerdo con la orientación estratégica del Hospital, puesto que no es clara para ellos. Durante la realización del cuestionario, se pudo observar que hay empleados que no hacen parte las estrategias desarrolladas por la Gerencia, con relación al cumplimiento de las metas y objetivos propuestos. En cuanto a las metas y objetivos, un $86 \%$ de los entrevistados manifestó trabajar eficientemente a favor del cumplimiento de las metas organizacionales a corto, mediano y largo plazo.

\section{Análisis del clima organizacional}

Frente a la dimensión de condiciones laborales, se puede observar lo siguiente:

\section{Figura No. 6. Resultados de la dimensión Condiciones Laborales.}

\section{Condiciones laborales}

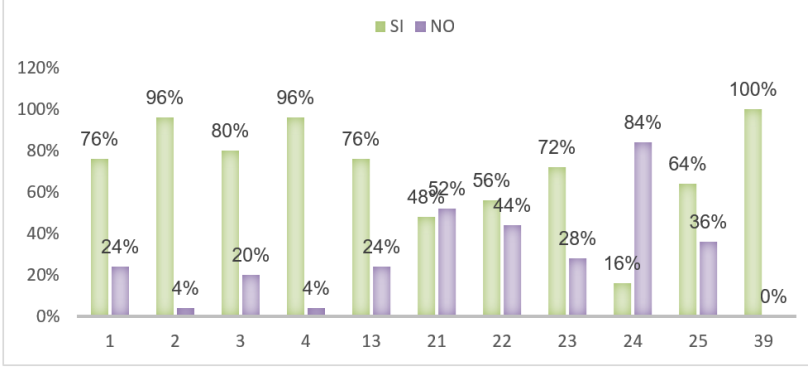

Fuente: Elaboración propia

La dimensión de condiciones laborales agrupa las variables de ambiente laboral. En cuanto a la satisfacción, se encontró que el $84 \%$ de empleados del Hospital manifiesta que tiene demasiada carga laboral, lo que les hace sentir que no tienen tiempo para descansar. Con relación a esto, el 52\% indica tener mucho trabajo y poco tiempo para realizar sus labores, pero al revisar detenidamente los porcentajes, se conoce que $100 \%$ del personal tiene claros los objetivos que fija el jefe inmediato con relación a cómo desarrollar durante la semana sus actividades, con el propósito de alcanzar las metas propuestas.

Para la segunda dimensión: Valoración, se destacan los siguientes aspectos: 
Figura No. 7. Resultados de la variable Valoración.

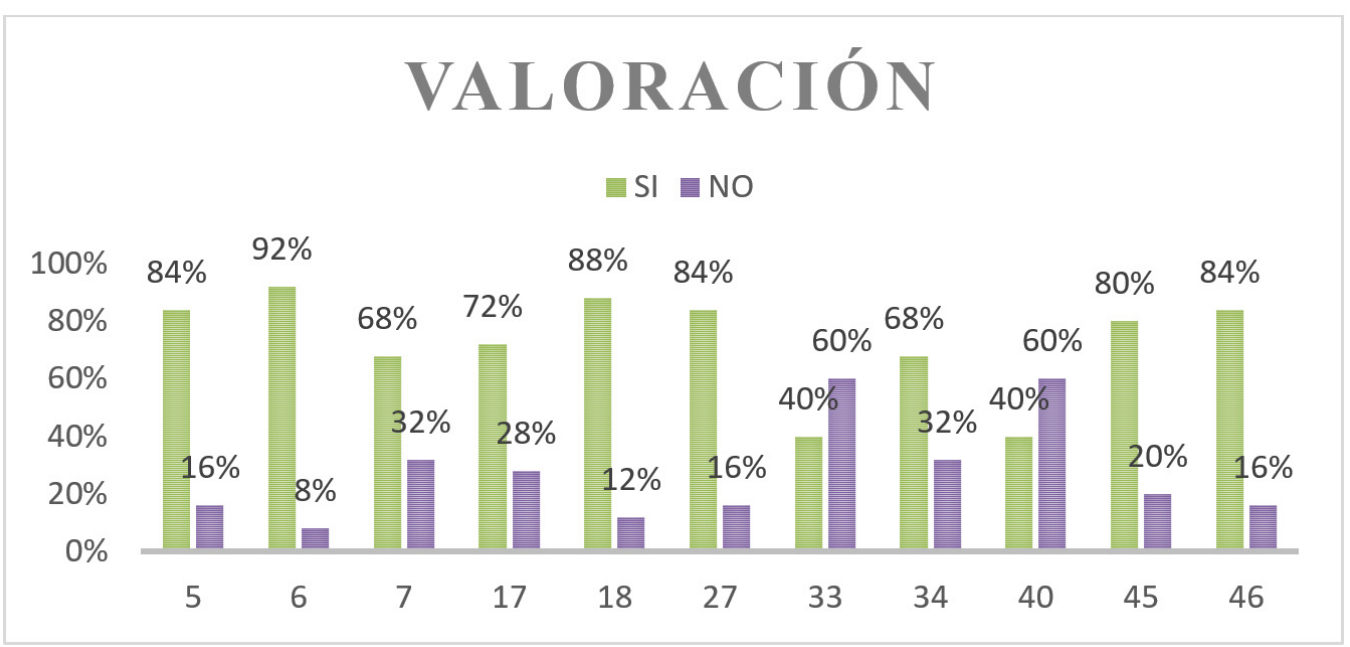

Fuente: Elaboración propia

Los resultados obtenidos en la dimensión de valoración del Hospital de Tabio muestran que, el 60\% de los entrevistados está en desacuerdo con la forma como se comunica la información, es decir, no hay un crecimiento laboral debido a que cuando hay una vacante disponible no se busca al posible candidato dentro de la organización, sino que se contrata a una persona externa.

En la dimensión de motivación, se puede evidenciar que:

Figura No. 8. Resultados de la variable Motivación.

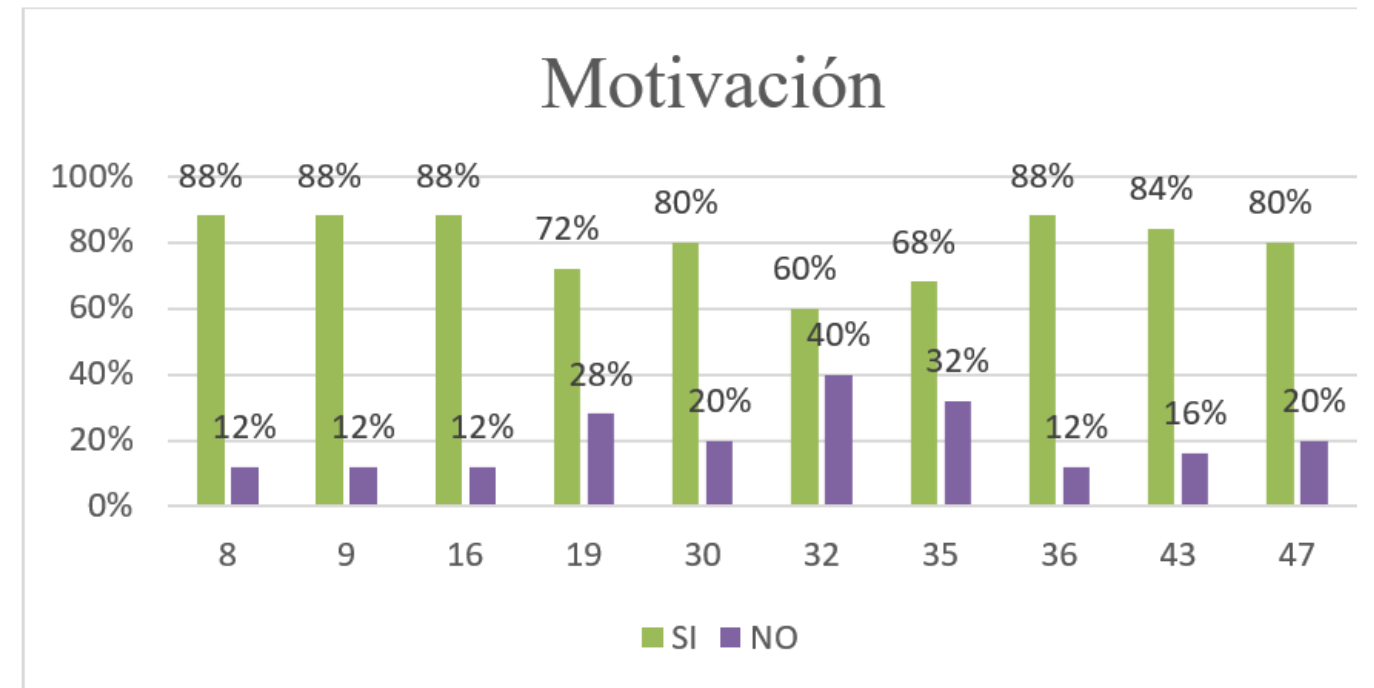

Fuente: Elaboración propia 
Es claro que, un empleado motivado es más productivo y eficiente al momento de alcanzar los objetivos o las metas de la organización. El Hospital cuenta con una serie de programas de motivación para sus empleados, dentro de los cuales se tiene desarrollo personal, reconocimiento por esfuerzos y aporte al logro de las metas trazadas; así, se alientan las ideas o aportes de los empleados, se fomenta un ambiente de innovación, y un plan de acción para los procesos de capacitaciones certificadas como un adicional a la hoja de vida de cada empleado. Sin embargo, una de las debilidades manifestada y observada, está relacionada con que los empleados no asisten a las capacitaciones por voluntad propia, sino que se impone la obligatoriedad.

En términos de la dimensión de responsabilidad, se manifiesta lo siguiente:

Figura No. 9. Resultados de la variable Responsabilidad.

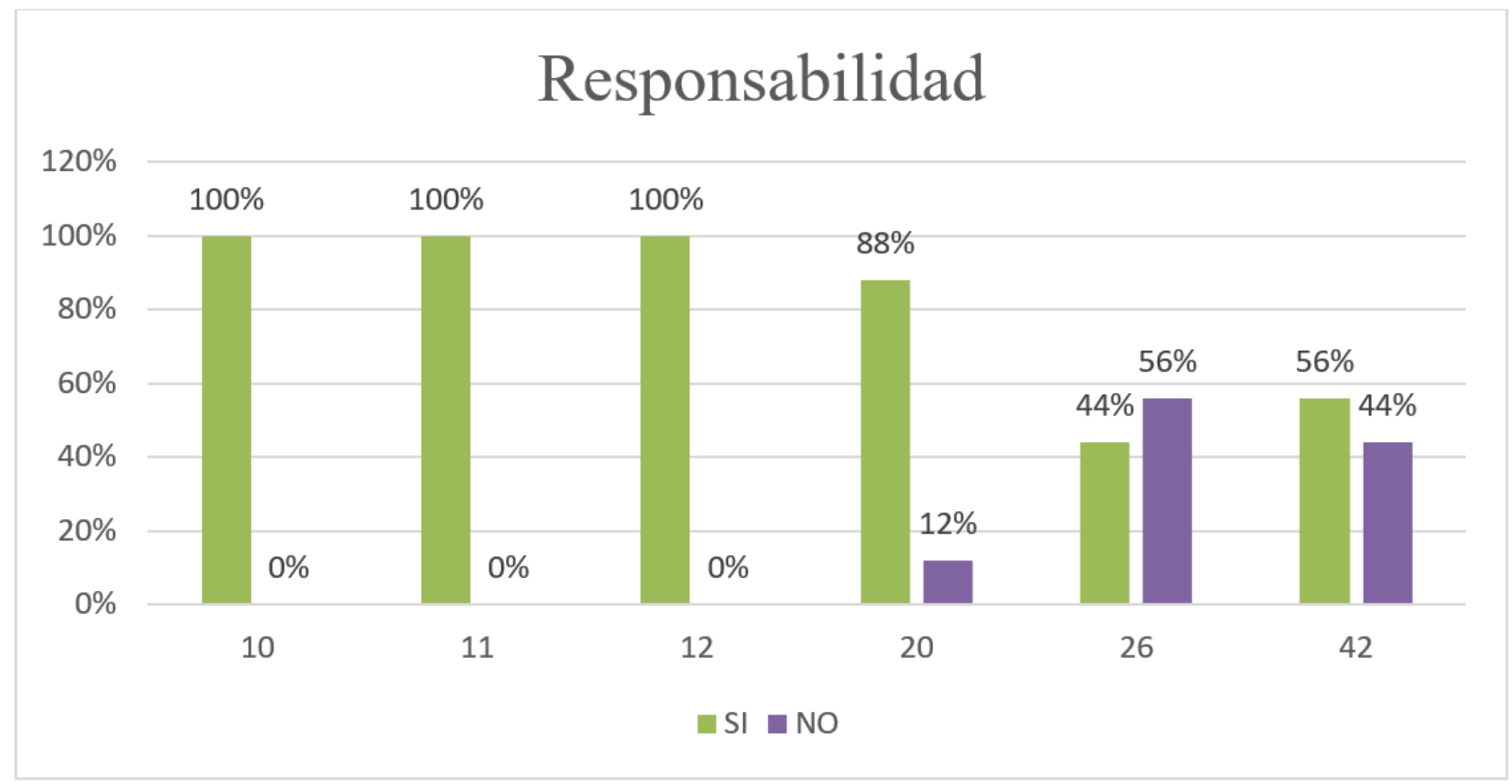

Fuente: Elaboración propia

En la dimensión de responsabilidad, hay aspectos positivos en los que todos los empleados están de acuerdo, es decir, se conoce que los estándares de desempeño y/o rendimientos, y la exigencia de cada puesto de trabajo dados los horarios de trabajo, permiten a los empleados atender necesidades personales. De esta manera, el $100 \%$ está de acuerdo con el supuesto de que su trabajo le permite realizar y cumplir los objetivos propuestos, mientras hay un $56 \%$ que no está de acuerdo en que para realizar su trabajo deba hacer un esfuerzo adicional, evitando su despido. Finalmente, cerca del $56 \%$ considera que el despido de un empleado se realiza por motivos propios de la persona, es decir, porque ha presentado dificultades para realizar sus labores dentro del Hospital.

Frente a la dimensión de trabajo en equipo, se evidencia que hay trabajo en equipo entre las áreas, se tiene buena comunicación con los compañeros, hay conciliaciones cuando hay un problema, se corrige a tiempo los errores y se evitan en el futuro. Sin embargo, en cuanto al trabajo entre diferentes equipos, se evidencian fallas en la comunicación. 


\section{Figura No. 10. Resultados de la variable Trabajo en Equipo.}

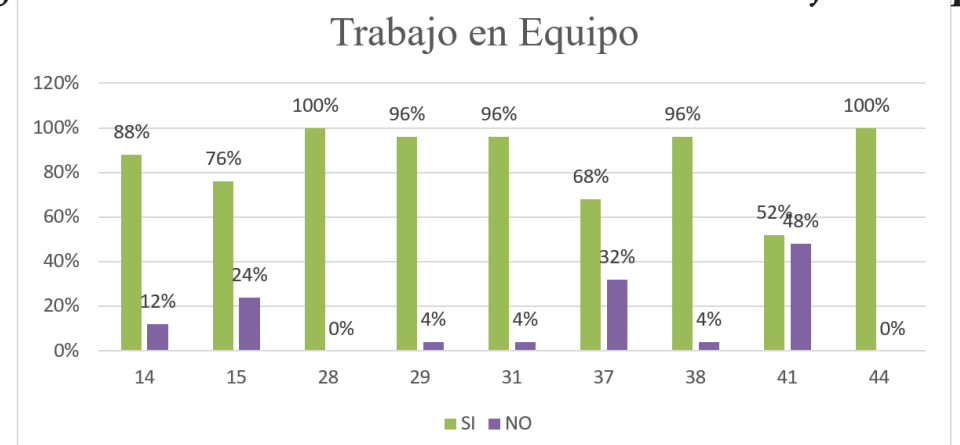

Fuente: Elaboración propia

\section{Conclusiones}

El modelo de gestión humana del Hospital de Tabio no es claro para los colaboradores y contratistas, lo que refleja poca motivación, fallas en la comunicación, carencia de liderazgo y un plan de formación inadecuado, impidiendo el cumplimiento de los objetivos de la organización.

Durante las diferentes actividades que se realizaron con los colaboradores de la empresa, ellos manifestaron su inconformidad frente a la forma de contratación. Actualmente, existen dos formas de vinculación: planta de colaboradores (nombramiento) o contratista (prestación de servicios), con cargas de trabajo similares, pero condiciones laborales muy diferentes. Lo anterior genera insatisfacción laboral y afecta de manera directa el funcionamiento del Hospital.

Así pues, se encontró que algunos colaboradores consideran que su labor es importante y que impacta de manera positiva el desarrollo de la organización, ya que aporta ideas orientadas a cumplir con los objetivos de su grupo. Sin embargo, las dificultades se evidencian en el momento de trabajar en equipo pues es ahí donde falla la comunicación, la objetividad, la convivencia y la optimización del tiempo; esta situación pone de manifiesto la importancia de diseñar un plan de fortalecimiento que permita liberarse de sentimientos negativos y aprender a convivir y desarrollar actividades juntos de manera más positiva.
El área de gestión humana manifestó que es difícil lograr la participación de todos los colaboradores en las distintas actividades de bienestar, tanto de formación como de socialización; por lo que muchos de los colaboradores desconocen los lineamientos estratégicos de la organización como misión, visión y objetivos, situación que obstaculiza que se alineen correctamente con ellos. Entonces, es fundamental para la organización diseñar un plan innovador para estas actividades, a partir de los gustos de sus colaboradores y motivarlos a participar activamente.

La variable implicación está relacionada con el trabajo en equipo y evidenció que, aún con dificultades, los equipos internos logran trabajar en equipo. No obstante, al relacionarse entre ellos, no se pueden llegar a acuerdos metodológicos, se pierde la comunicación y no se encuentran estrategias para el cumplimiento de las metas y objetivos propuestos.

En la variable consistencia, se puede concluir que cada equipo de trabajo tiene identificados a sus líderes, formas de trabajo, capacidades y habilidades; pero que al trabajar con otras áreas se confunden los liderazgos y metodologías; lo que se dificulta aún más por la falta de colaboración y proactividad de algunos colaboradores, en especial los de planta, que se limitan solo a hacer la función para la que fueron contratados sin disponerse a apoyar en otras labores. 
Con respecto a la variable adaptación, se observó que a pesar que los colaboradores suelen dar ideas para innovar y mejorar procesos y procedimientos; estas no son tenidas en cuenta por los directivos de la organización, lo que ocasiona desinterés y desmotivación. En la variable de misión, se logró evidenciar que los colaboradores no tienen clara la orientación estratégica general del Hospital, ni la implementada por cada líder de equipo, debido a que carecen de una comunicación asertiva y control de la información; es decir, que la información se va perdiendo o confundiendo en el camino; lo que genera fallas en el cumplimiento de los objetivos y ocasiona un caldo de cultivo para el chisme y la informalidad.

$\mathrm{Al}$ analizar cada variable de forma independiente, se concluye que, si bien el Hospital presenta puntos críticos de comunicación, trabajo en equipo y liderazgo; es consciente de ello y está comprometido con un plan de mejoramiento. Por ejemplo, se habla de una cultura organizacional con principios, valores, normas; con un clima adecuado donde se muestra cómo es el individuo en la organización, su forma de relacionarse, llevar a cabo sus labores y adaptarse a las condiciones del entorno.

Las condiciones laborales se relacionan con los puestos de trabajo, los materiales y recursos utilizados por cada empleado para realizar sus labores. Así, se evidencia que, a pesar de contar con los recursos físicos adecuados, los empleados sienten que tienen sobrecarga laboral, lo que genera estrés, cansancio y desmotivación. Con respeto a la dimensión valoración, queda claro que el Hospital no cuenta con un sistema de motivación que resalte y valore el trabajo de las personas; lo que las lleva a sentir que no son tenidas en cuenta y las desmotiva para participar en las diferentes actividades de formación y bienestar a las que asisten como un requisito laboral. También es fundamental para el Hospital diseñar un plan adecuado de valoración y reconocimiento, tanto del personal de planta como de los contratistas, en la que se reconozcan sus habilidades y aportes al crecimiento de la organización.

En la dimensión responsabilidades se evidenció que los empleados sienten sobrecarga laboral, pero a la vez no están dispuestos a dar más de sí mismos para lograr hacer sus labores a tiempo. Lo anterior, se evidencia con la política de rotar el personal de una zona a otra, puesto que esto genera inconvenientes. En cuanto a la dimensión de trabajo en equipo, se puede concluir que es uno de los puntos más vulnerables para los colaboradores, puesto que a pesar de la autoridad y respeto hacia los líderes de cada área, existen inconvenientes por la rotación de personal que, además genera alta resistencia al cambio.

Por último, es importante decir que, los empleados de Hospital de Tabio requieren de un programa de sensibilización y motivación frente a mayor sentido de pertenencia con la entidad. La investigación de la Universidad de Cundinamarca sugirió el diseño de un Programa de Endomarketing para el Hospital, propuesta que fue aceptada y cuyos resultados fueron presentados como parte de otros productos de la investigación en Gestión del Talento Humano.

\section{Referencias bibliográficas}

Bonavida y Prado (2010). Adaptación al español del instrumento sobre cultura organizacional de Denison. SUMMA Psicológica UST.

Denison, D. R. y Neale, W. (2000). Denison Organizational Culture Survey. Ann Arbor: Denison Consulting. 